\title{
$-5$
}

NASA Technical Memorandum 105280

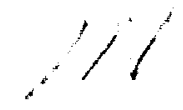

$45 \% 56$

P-17

\section{Theoretical Modelling of AFM for Bimetallic Tip-Substrate Interactions}

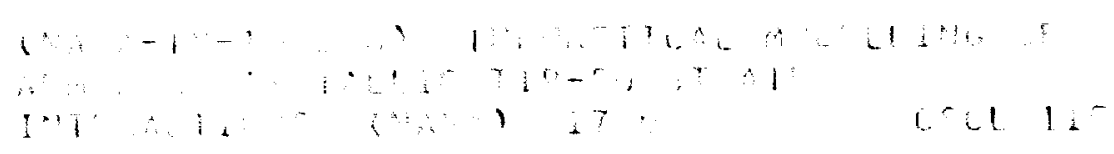

$$
36 \quad \text { inclis }
$$

Guillermo Bozzolo

Analex Corporation

Brook Park, Ohio

and

John Ferrante

Lewis Research Center

Cleveland, Ohio

October 1991 



\title{
THEORETICAL MODELLING OF AFM FOR BIMETALLIC \\ TIP-SUBSTRATE INTERACTIONS \\ Guillermo Bozzolo \\ Analex Corporation \\ 3001 Aerospace Parkway \\ Brook Park, Ohio 44142-1003 \\ John Ferrante \\ National Aeronautics and Space Administration \\ Lewis Research Center \\ Cleveland, Ohio 44135-3191
}

\begin{abstract}
Recently, a new technique for calculating the defect energetics of alloys based on Equivalent Crystal Theory has been developed. This new technique successfully predicts the bulk properties for binary alloys as well as segregation energies in the dilute limit. We apply this method for the calculation of energy and force as a function of separation of an AFM tip and substrate. This study was done for different combinations of tip and sample materials. The validity of the universality discovered for same metal interfaces is examined for the case of different metal interactions.
\end{abstract}


The atomic force microscope (AFM) [1] has enabled the measurement of interfacial forces at the atomic level as a function of separation [2]. However, the ability to perform theoretical calculations of these forces is limited by the complexity of tip geometries. Also, the need to calculate force as a function of separation and the need to include relaxation makes first-principles calculations difficult to perform. A new formalism, Equivalent Crystal Theory (ECT) [3], which has given accurate surface energies and relaxations for fcc, bcc metals and semiconductors [4] has been used to calculate the force versus separation for a range of tip geometries [5]. However, ECT has only been formulated for single component materials, whereas it is necessary to be able to calculate the same properties for different materials in contact.

Recently, a new method for treating multicomponent systems, which builds on the ideas of ECT, was introduced by Bozzolo, Ferrante and Smith (BFS) [6]. Here, we give a brief description of the method and present an application to a number of fcc and bcc metals in contact for several tip geometries. We will present the calculations of the binding energy and force as a function of separation and discuss the universal aspects of these curves.

In BFS, the total energy of a multicomponent system is the sum of the energies of individual atoms. For each atom, we break up the energy into two parts: a strain energy $e^{S}$ and a chemical contribution $e^{C}$, linked by a coupling factor $g$ :

$$
e_{i}=e_{i}^{S}+g_{i}\left(e_{i}^{C}-e_{0_{i}}^{C}\right)
$$

where $i$ denotes the atomic species of a given atom.

The strain energy, $e_{i}^{S}$, is obtained from a standard ECT defect calculation where all the atoms surrounding the atom in question are taken to be of the same atomic species $i$. 
In the spirit of ECT, an equivalent lattice parameter $\boldsymbol{a}_{i}^{S}$ is straightforwardly computed and the strain contribution becomes

$$
e_{i}^{S}=E_{C}^{i} F^{*}\left(a_{i}^{S *}\right)
$$

where $F^{*}(a)=1-(1+a) e^{-a}$ and $E_{C}^{i}$ is the cohesive energy of an atom of species $i$. The argument $a_{i}^{S *}$ is given by $a_{i}^{S *}=\left(a_{i}^{S}-a_{e}^{i}\right) / h_{i}$, where $a_{e}^{i}$ and $L_{i}$ are the lattice parameter and screening length of a pure crystal of species $i$. The coupling term $g_{i}$ is defined in terms of the scaled lattice parameter $a_{i}^{S *}$ as $g_{i}=\exp \left(-a_{i}^{S_{*}}\right)$.

The chemical contribution $e_{i}^{C}$ is obtained by yet another standard ECT calculation. As opposed to the strain energy term, the surrounding atoms retain their chemical identity, but are forced to be in equilibrium lattice sites. If $N_{i k}\left(M_{i k}\right)$ denotes the number of nearest(next)-neighbors of species $k$ of the atom in question (of species $i$ ) then the ECT equation [4] to be solved for the equivalent lattice parameter $a_{i}^{C}$ is

$$
N R_{1}^{p_{i}} e^{-c_{i} R_{1}}+M R_{2}^{p_{i}} e^{-\left(\alpha_{i}+\frac{1}{\lambda_{i}}\right) R_{2}}=\sum_{k} N_{i k} r_{1}^{p_{i}} e^{-\alpha_{i k} r_{1}}+\sum_{k} M_{i k} \tau_{2}^{p_{i}} e^{-\left(\alpha_{i k}+\frac{1}{\lambda_{i}}\right) r_{2}}
$$

where $N(M)$ is the number of nearest(next)-neighbors in the equivalent crystal of species $i$ and $R_{1}\left(R_{2}\right)$ is the nearest(next)-neighbor distance in the equivalent crystal of lattice parameter $a_{i}^{C}, r_{1}$ and $r_{2}$, are the equilibrium nearest- and next-nearest-neighbor distances in an equilibrium crystal of species $i$, respectively. The chemical energy is then computed with

$$
e_{i}^{C}=\gamma E_{C}^{i} F^{*}\left(a_{i}^{C *}\right)
$$

and

$$
e_{0_{i}}^{C}=\gamma_{0} E_{C}^{i} F^{*}\left(a_{0}^{C *}\right)
$$


where $\gamma\left(\gamma_{0}\right)=+1$ if $a_{i}^{C *}\left(a_{0_{i}}^{C *}\right) \geq 0$ and $\gamma\left(\gamma_{0}\right)=-1$ otherwise, and $a_{i}^{C *}=\left(a_{i}^{C}-a_{e}^{i}\right) / h_{i}$. The scaled lattice parameter $a_{i}^{C *}$ is obtained from Eq.(3) with the parameters $\alpha_{i k}$ listed in Ref. 6 , and $a_{0_{i}}^{C *}$ is computed by solving Eq.(3) but with $\alpha_{i k}=\alpha_{i}$. The rest of the parameters appearing in Eq.(3) are listed in Ref. 6.

An overview of typical results is shown in Figs. 1-3. Fig. 1 displays the comparison between the experimental values of the heat of formation [7] of selected binary compounds $A_{x} B_{1-x}$ and the predictions of this method, for the full range of concentration $x$. In Fig. 2 we compare the predictions for the lattice parameters of the binary alloys of $\mathrm{Ni}, \mathrm{Cu}$ and $\mathrm{Pd}$ with experimental values [8]. The method's sensitivity is highlighted in Fig. 3, where the lattice parameters of $\mathrm{Ag}-\mathrm{Au}$ alloys are compared with experiment [8]. A lattice contraction is experimentally detected for certain concentrations involving significantly small changes of the lattice spacing, and they are successfully reproduced by our method. Similar results are obtained for bcc alloys. A full description of the method is given in Ref. 6. Even though BFS is a semiempirical method, its dependence on experimental input is minimal in that only two experimental values (i.e., the heats of solution in the dilute limit) are needed and most of the formalism is based on the behavior of the pure elements.

The only limitation is the availability of reliable experimental data for the corresponding alloys. BFS is designed to provide, with minimum computational effort, a systematic description of arbitrary systems of more than one atomic species. Because of its numerical simplicity, this method, together with a Monte Carlo procedure to account for individual atomic displacements, is particularly well suited for computer simulations of typical STM/AFM experiments.

In this first application of BFS to the study of metallic adhesion using AFM, we consid- 
ered several bimetallic junctions, concentrating on the effect of the tip shape on the adhesive forces in AFM. We left out the possibility of individual atomic displacements and avalanche effects for small gap widths, as our concern is with the existence of a universal behavior that include bimetallic systems in ideal (i.e., rigid) conditions. If these effects were taken into account, one would expect a greater sensitivity of the energetics of the tip geometry as well as tip and sample materials. We restricted our calculations to systems in which both the sample and the tip are fcc or bcc metals. Although there is some experimental evidence that multiple atom tips are typical in the scanning process [10], in this initial study we choose to model the interaction between the tip and the substrate with a single-atom tip. We will show that different tip geometries account for minor corrections to the energetics of the system. This result is consistent with the body of available STM/AFM data [10].

Fig. 4.a shows the variation of binding energy with separation for two different STM/AFM Al tips on a (100) Al sample. The tip-substrate separation is taken to be zero when the tip atom is in contact with the sample surface (in this calculation, this corresponds to the fcc-hollow site). The two tips examined are sketched in Figs. 4.b and 4.c. As discussed before, the differences in energy due to different tip geometries are rather small. Moreover, the adhesive energy (i.e., the minimum of the binding energy curve), if amenable to measurement, should give an indication of the tip shape.

Fig. 5 displays the dependence of the binding energy curve in terms of lattice symmetries. Two different surfaces of Ir are considered: (100) and (111). As expected, the adhesive energy values are smaller for the (111) face. These curves correspond to a pyramidal tip (see Fig. 4.c), but the essential features should be the same for other tip shapes.

Fig. 6.a and 6.b summarize the binding energy versus separation results for a variety 
of fcc and bcc materials ( $\mathrm{Al}, \mathrm{Ni}, \mathrm{Cr}, \mathrm{Ir}$ ) for their (100) face and a pyramidal tip. Consistent with previous findings, all curves scale onto one curve which is accurately described by a Rydberg function. Similar conclusions can be drawn for the force vs. separation curves, shown in Fig. 6.c.

Finally, Fig. 7 displays the variation of binding energy with separation for a variety of tip-substrate systems A-B (A:tip-B:substrate) where Ni is one of the components. There is a noticeable change in the qualitative behavior of these results as the lattice parameter mismatch between the components changes. However, we found that for bimetallic junctions, the binding energy curves also scale onto a unique curve which can be fitted satisfactorily Rydberg function, as in the case of pure metals as shown in Fig. 7.d. This result is independent of lattice structure, crystal face, tip geometry and materials involved.

Simulations like the ones presented in this work are computationally extremely simple, allowing for straightforward extensions of these ideal situations, including multiatomic tip effects and, more importantly, tip induced relaxations. In particular, BFS, together with the recently developed theory of avalanche in adhesion [11], should be ideally suited for the study of stability of a tip and sample at very small separations [12].

\section{Conclusions}

The semiempirical method for the treatment of multicomponent systems developed by Bozzolo, Ferrante and Smith is physically accurate and computationally simple. Because of the ability of this method to effectively deal with systems with more than one atomic species, it provides an ideal framework for theoretical modelling of defect structures such as impurity atoms. For the case of STM/AFM, applications to a variety of tip-substrate combinations, together with some insight on the dependence of the tip structure yield encouraging results, 
in the sense that in all the situations considered, the universal behavior expected (and observed) for pure metals is also reproduced. 


\section{References ,}

${ }^{1}$ G. Binnig, C.F. Quate and Ch. Gerber, Phys. Rev. Lett. 56 (1986) 930.

${ }^{2}$ U. Dürig, O. Züger and D.W. Pohl, Phys. Rev. Lett. 65 (1990) 349.; ibid., J. of Microscopy 152 (1988) 259.

3John R. Smith and Amitava Banerjea, Phys. Rev. Lett. 59 (1987) 2451

4John R. Smith and Amitava Banerjea, Phys. Rev. B37 (1988) 10411; John R. Smith, Tom Perry, Amitava Banerjea, John Ferrante and Guillermo Bozzolo, Phys. Rev. B (in press)

${ }^{5}$ A. Banerjea, John R. Smith and John Ferrante, J. Phys.: Condens. Matter 1 (1989) 9823.

${ }^{6}$ Guillermo Bozzolo and John Ferrante, N.A.S.A. Technical Publication (1991) (in press); Guillermo Bozzolo, John Ferrante and John R. Smith (to be published)

${ }^{7}$ R. Hultgren, R. L. Orr, P. D. Anderson and K. K. Kelley in Selected Values of the Thermodynamic Properties of Binary Alloys (Wiley, N.Y., 1963).

${ }^{8}$ W. B. Pearson in Handbook of Lattice Spacings and Structures of Metals (Pergamon Press, 1967).

9James H. Rose, John Ferrante and John R. Smith, Phys.Rev.Lett. 47 (1981) 675.

${ }^{10}$ Farid F. Abraham, Inder P. Batra and S. Ciraci, Phys. Rev. Lett. 60 (1988) 1314; Farid F. Abraham and Inder P. Batra, Surf. Sci. Lett. 209 (1989) 125; D. Tománek, G. Overney, H. Miyazaki, S.D. Mahanti and H. J. Güntherodt, Phys. Rev. Lett. 63 (1989) 876; E. Tekman and S. Ciraci, J. Phys.: Condens. Matter 3 (1991) 2613 and references therein.

${ }^{11}$ John R. Smith, Guillermo Bozzolo, Amitava Banerjea and John Ferrante, Phys. Rev. Lett. 63 (1989) 1269.

${ }^{12}$ J.B. Pethica and A. P. Sutton, J. Vac. Sci. Technol. A6 (1988) 2490. 


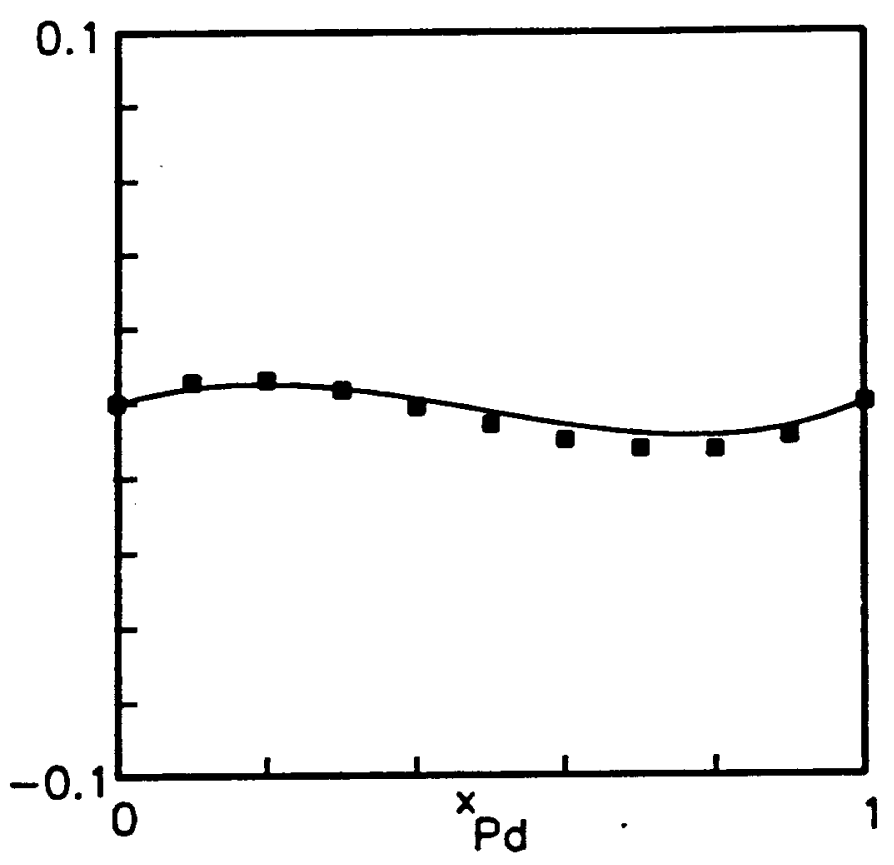

(a) Pd-NI.

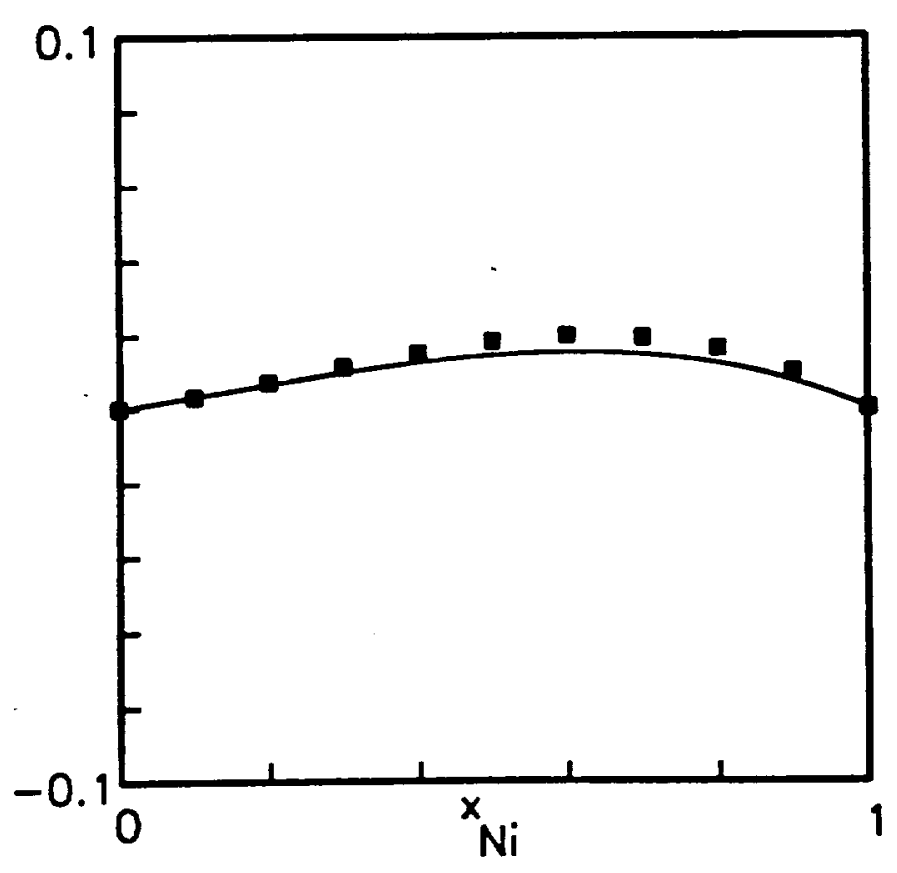

(c) Ni-Cu.

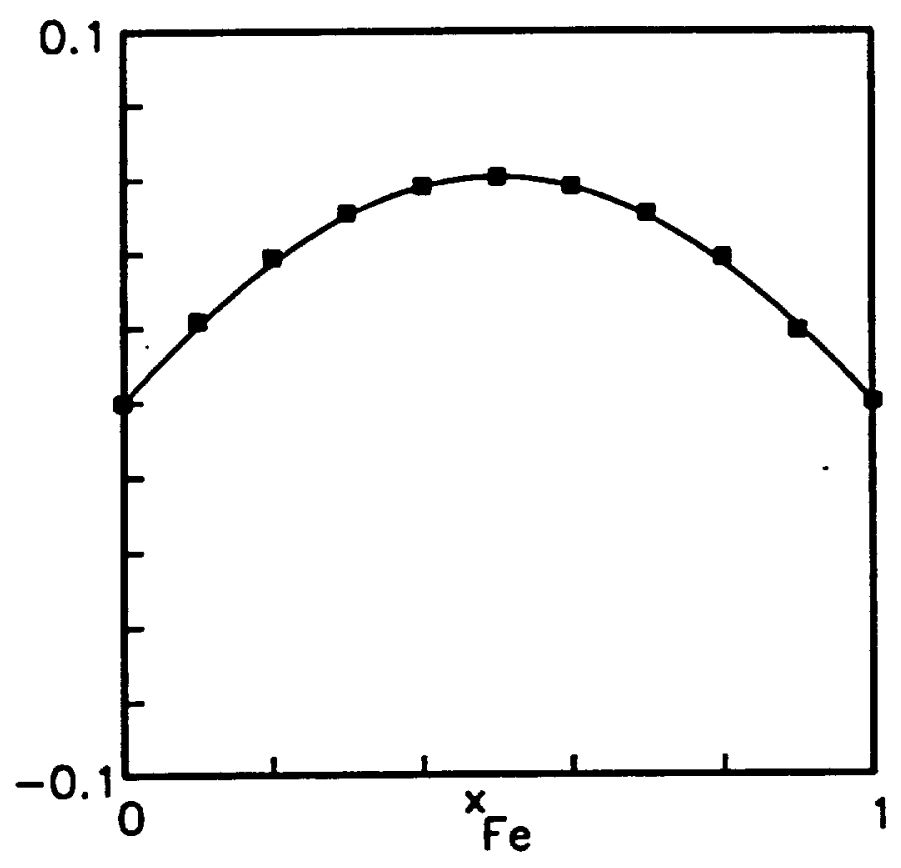

(b) Fo-Cr.

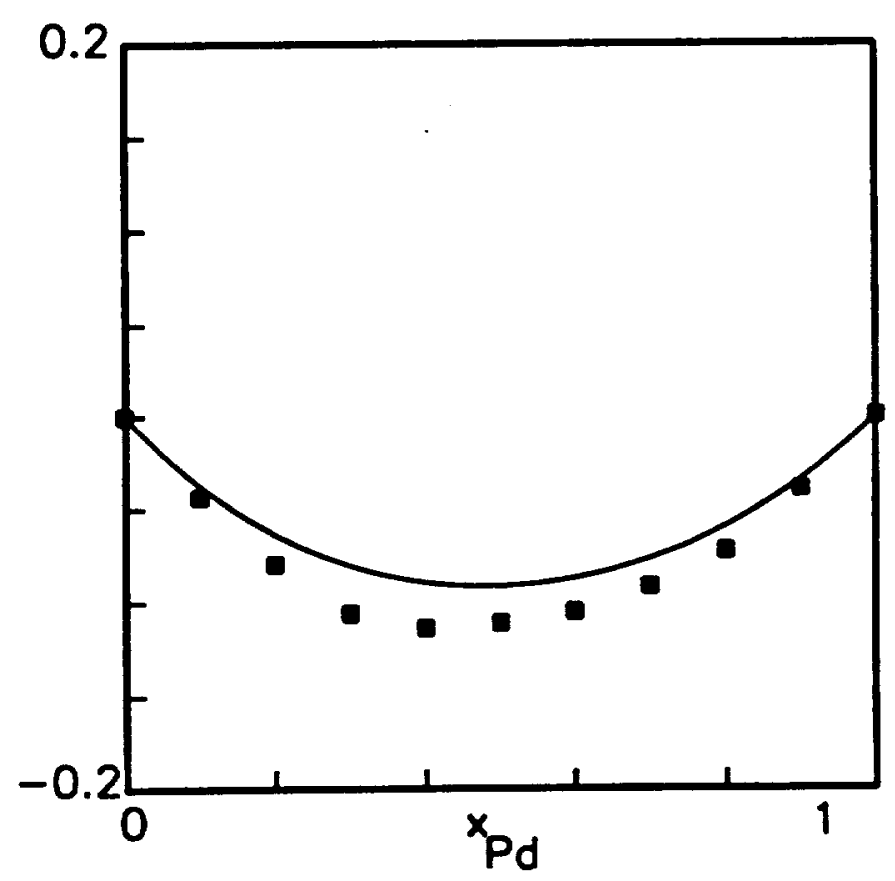

(d) Pd-Cu.

Figure 1.-Comparison between the heat of formation as a function of concentration for alloys the solld curve indicates the results obtained with BFS, ant the dots indicate experimental values. The heat of formation is in cal $\mathbf{g}$-atom. 


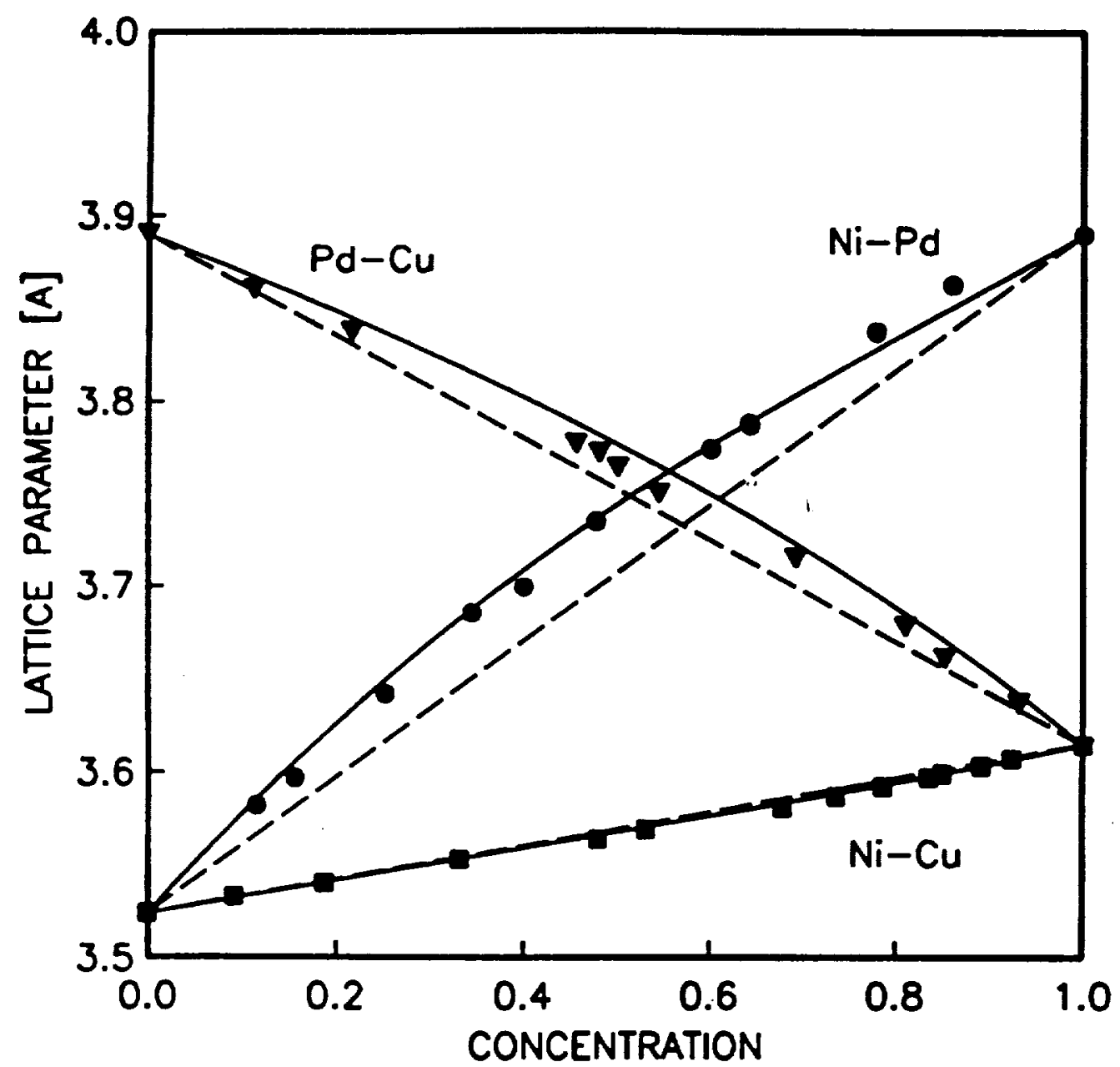

Figure 2.-Compatson between the lattice parameters obtained with BFS (colld curve) and Vegard's law results (dashed lines) for different fcc binary alloys. Experimental velues are shown for NI-Cu (solid squares), NiPd (solld clrcles) and Pd-Cu (solld triangles). 


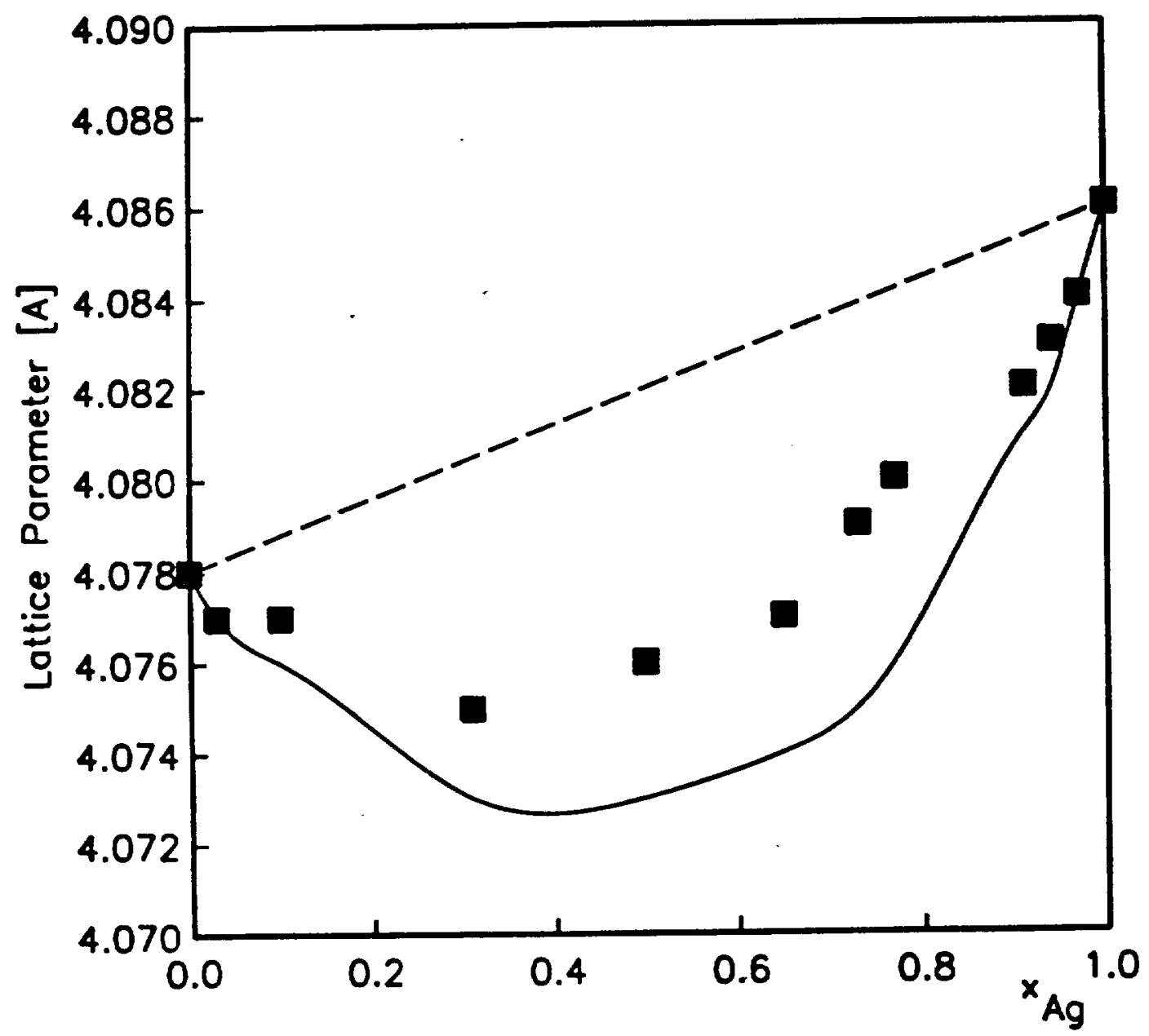

Figure 3. - Comparison between the results obtained with BFS (solid curve) and experimental values (solid squares) for the lattice parameters of Ag-Au alloys as a function of comparison. Vegard's law results are also shown (dashed line). 


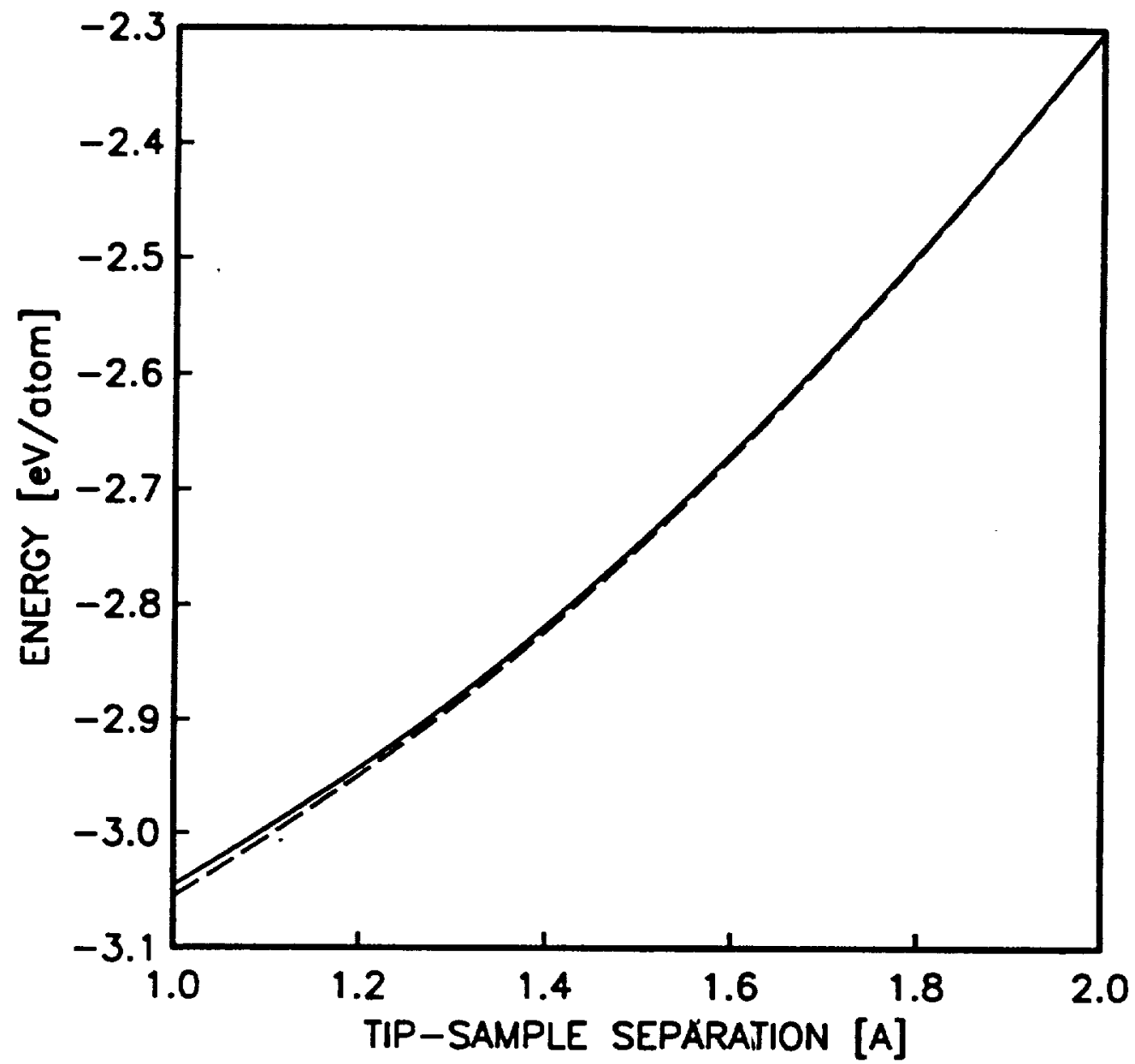

(a) Variation of binding energy with separation for a STMAFM A tip at the fCc-hollow stle of a N (100) surface for a one-atom tip (solid curve) and a pyramidel tip (dashed curve). The separniton bo taken to be zero when tip and substrate are in contact.

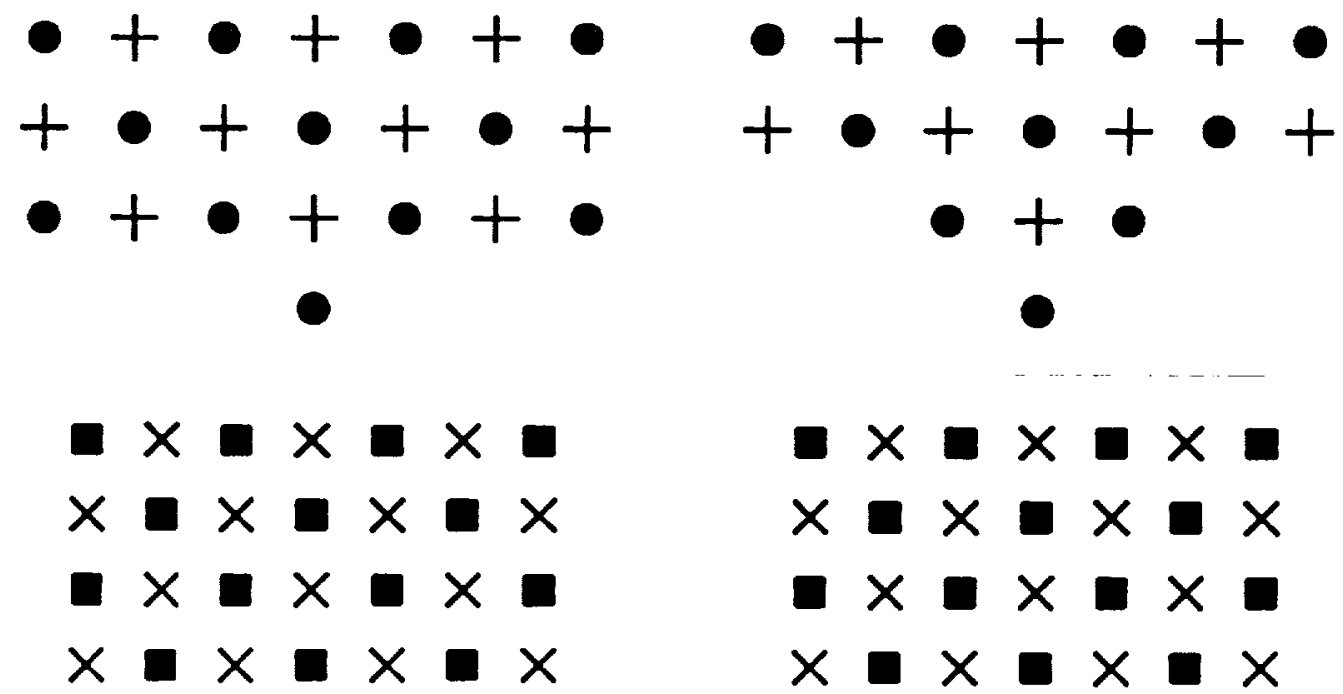

(b) One-atom tip diegram of a (100) tace fcc metal. For the substrate, square dots indicate atoms on the plane of the page while the crosses $(x)$ indicate parallel planes below or above the plane of the page. Solld clrcles and $(+)$ are used for the tip.

Figure 4.

(c) Pyramidal tip diagram of a $(100)$ face fcc metal. 


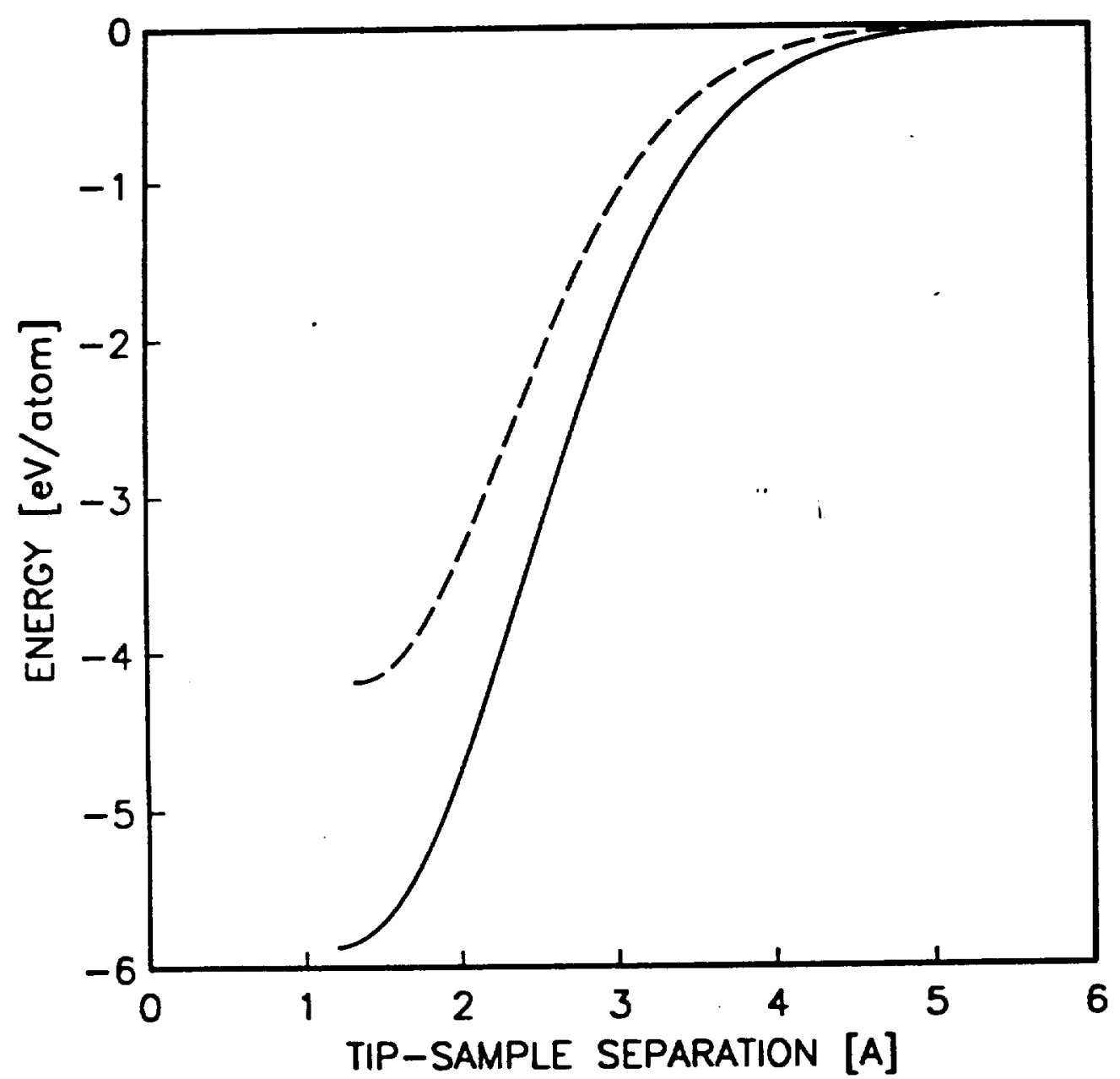

Figure 5.-Variation of binding energy with separation for an Ir tip on a (100) surface (solid curve) and (111) (deshed curve) Ir surface. The separation is taken to be zero when tip and substrate are in contact. 


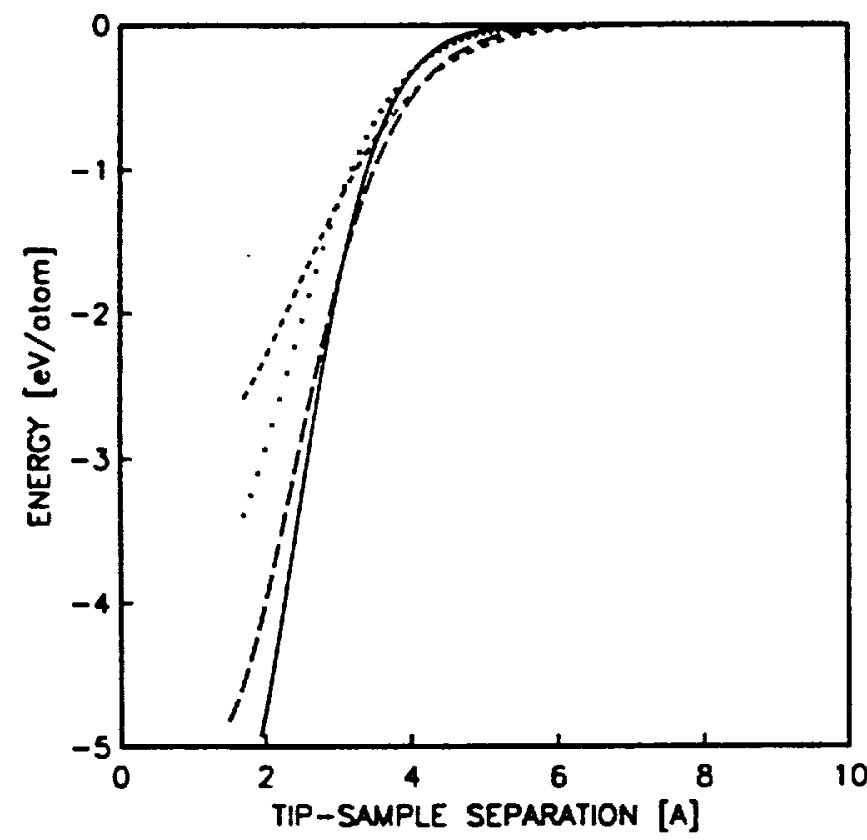

(a) Veriation of binding energy with separation at the hollow alte on - (100) surface of different foc and boc metals: AVAl (short desh), NWNI (dots), Cr/Cr (tong dash), Ir/H (solld curve).

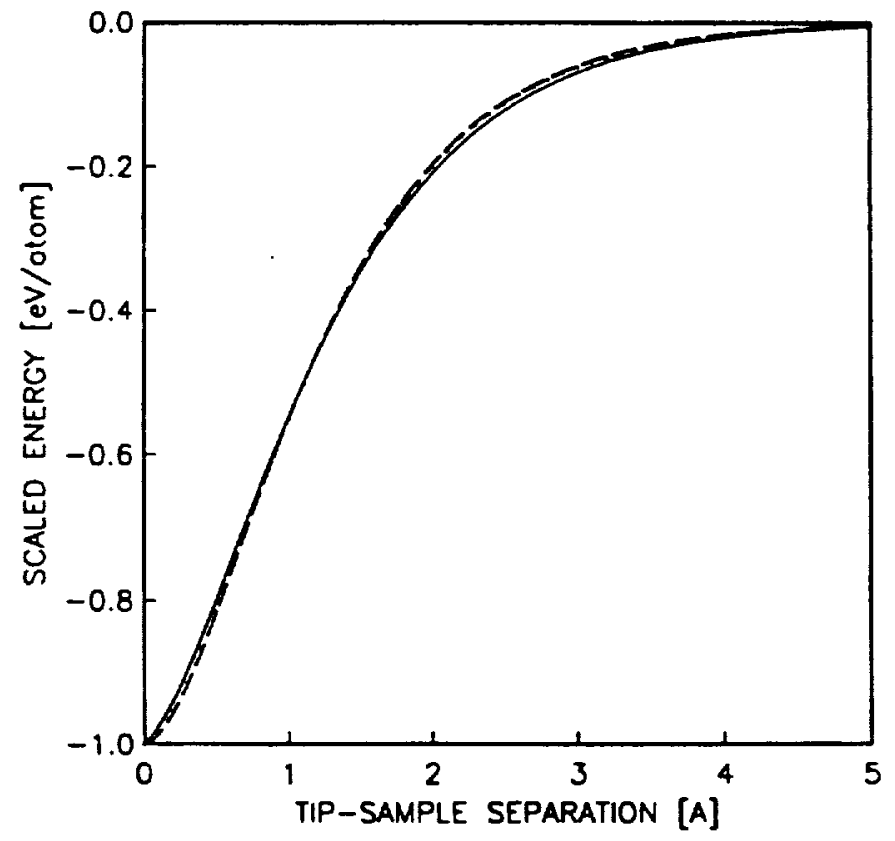

(b) Scaled binding energlose and separations fot the cease shown in (a).

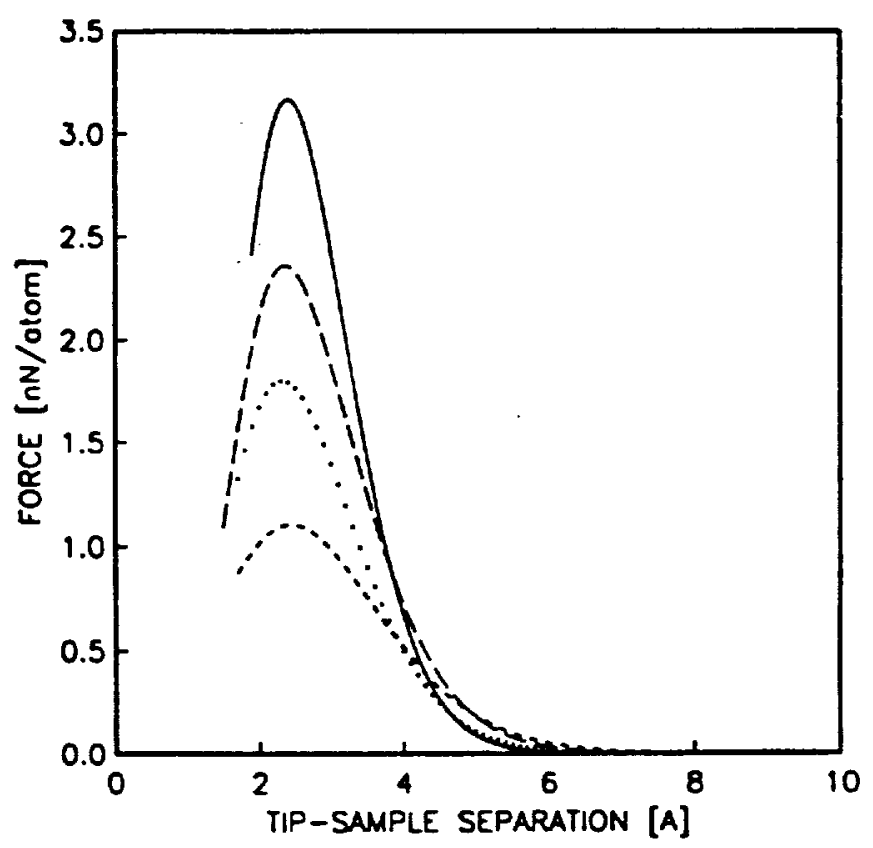

(c) Variation of the force with separation for the cases shown in (a).

Figure 6. 


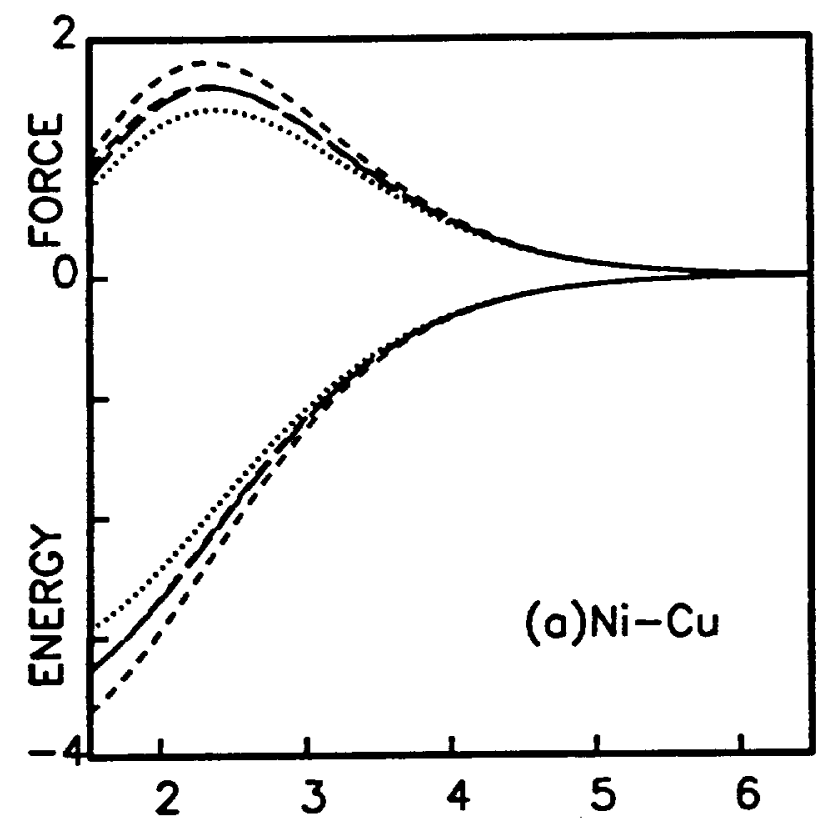

(a) NWNI (solld curve), NVCu (tong dash), CWNI (short dash), cuvcu (dottod curve).

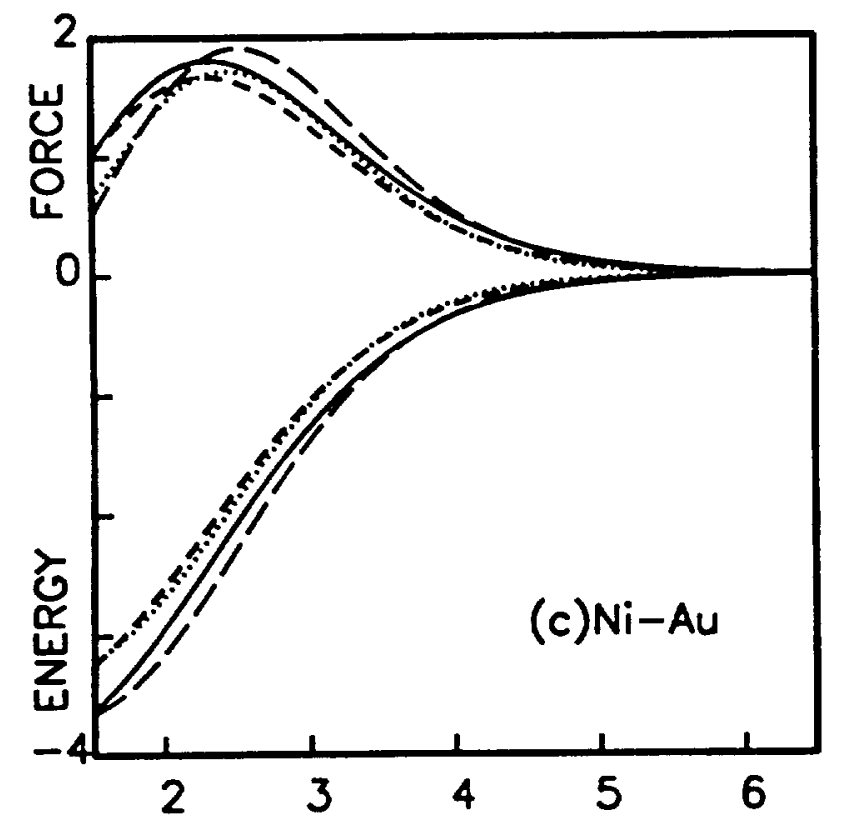

(c) NVNI, NVAU, AUNI and AWAU.

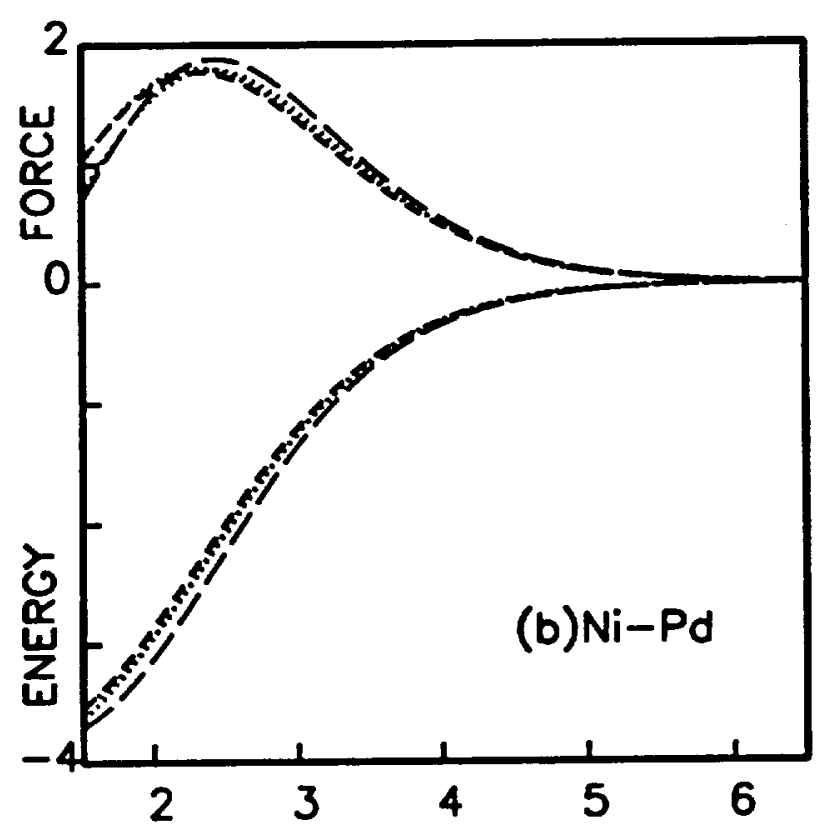

(b) NWI, NUPd, Pd/Ni and Pd/Pd.

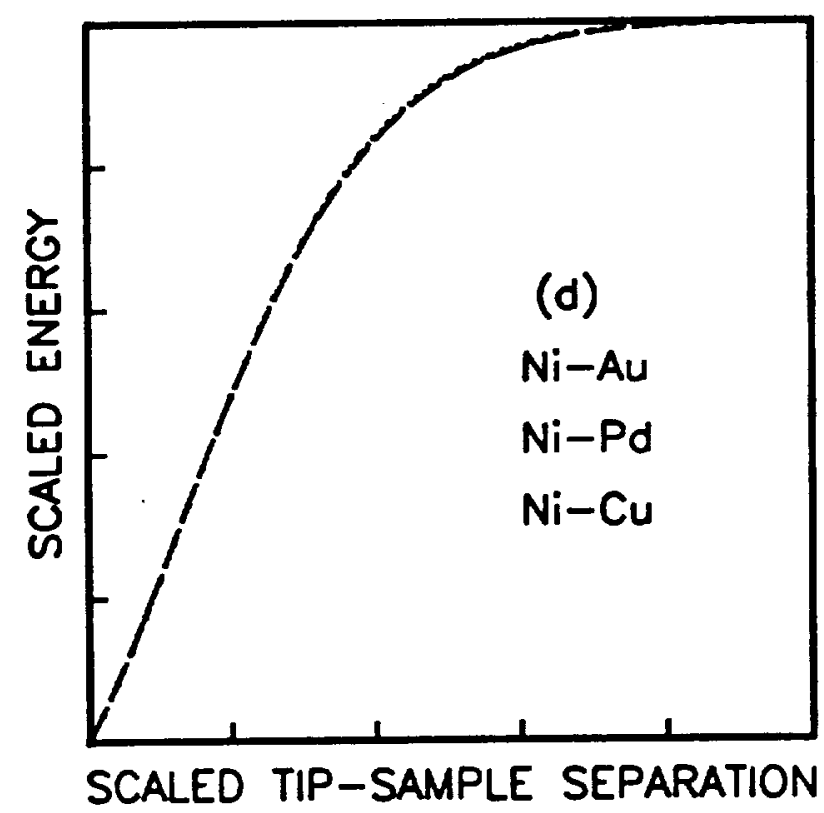

(d) Scaled binding energles and separations for the cases shown in (a), (b), (c).

Figure 7.-Variation of binding energy and force with seperation for different bimetallic junctions A-B (Attip-B:substrate). 


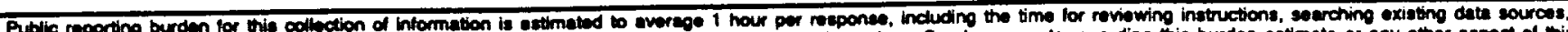

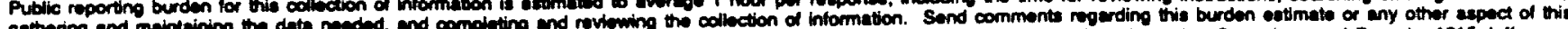

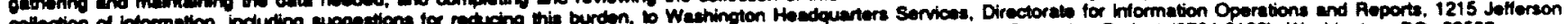

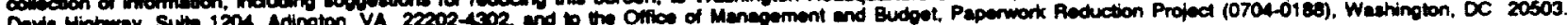

\begin{tabular}{l|l|l|} 
1. ACENCY USE ONLY (Leave blank) & 2. REPOAT DATE & 3. REPORT TYPE AND DATES COVERED
\end{tabular}

\begin{tabular}{l|r|r|r|r} 
October 1991 & Technical Memorandum
\end{tabular}

4. TITLE AND SUBTTLE

Theoretical Modelling of AFM for Bimetallic Tip-Substrate Interactions

6. AUTHOR(S)

Guillermo Bozzolo and John Ferrante
5. FUNDING NUMBERS

WU-505-90-52

- performino oroanization REPORT NUMBER

E-6585

10. SPONSORINGMONITORINO AOENCY REPORT NUMBEA

NASA TM- 105280

National Aeronautics and Space Administration

Washington, D.C. 20546-0001

NASA TM-10S280

11. SUPPLEMENTARY NOTES

Guillermo Bozzolo, Analex Corporation, 3001 Aerospace Parkway, Brook Park, Ohio 44142; John Ferrante, NASA Lewis

Research Center. Responsible person, John Ferrante, (216) 433-6069.

12. DISTAIBUTION/AVAILABILITY STATEMENT

12b. DISTRIBUTION CODE

Uniclassified-Unlimited

Subject Category 26

13. ABSTAACT (Maximum 200 words)

Recently, a new technique for calculating the defect energetics of alloys based on Equivalent Crystal Theory has been developed. This new technique successfully predicts the bulk properties for binary alloys as well as segregation energies in the dilute limit. We apply this method for the calculation of energy and force as a function of separation of an AFM tip and substrate. This study was done for different combinations of tip and sample materials. The validity of the universality discovered for same metal interfaces is examined for the case of different metal interactions.

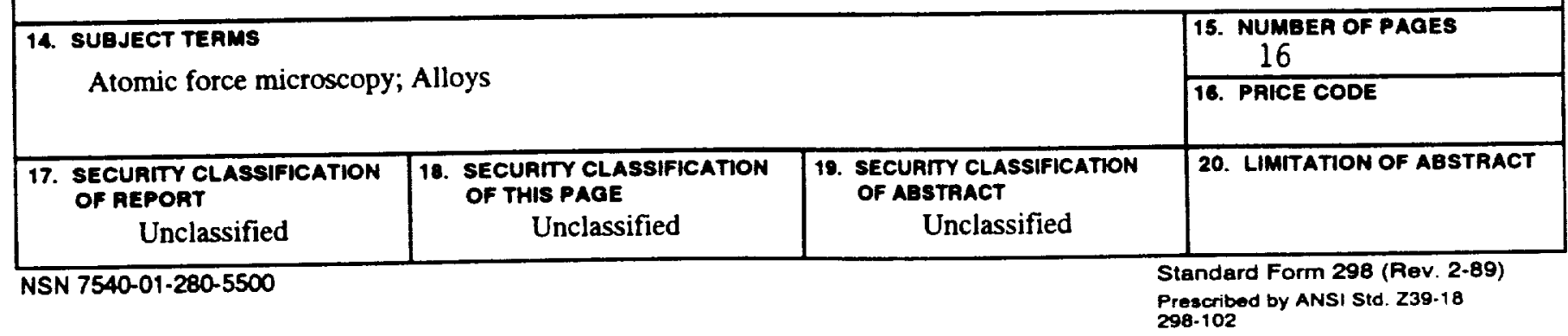



National Aeronautics and Space Administration

Lewis Research Conter

Cleveland, Ohio 44135

Omelel Buelnew

Penatry for Privete Ulas 2500
FOUATH CLASB MARL

\section{ADORESS COARECTION REQUESTED}

|||| $\mid$

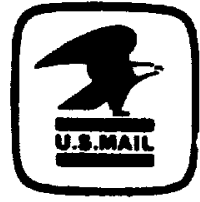

Posiage and F ees Pard

National Aeronaulics and

Space Adminisiration

NASA 451 